\title{
ATLAS DETECTOR OVERVIEW (OPERATION EXPERIENCE, PERFORMANCE)
}

\author{
ANA HENRIQUES \\ On behalf of the ATLAS collaboration \\ CERN, Switzerland (Ana.Henriques@cern.ch)
}

\begin{abstract}
ATLAS is a general-purpose detector located at one of the 4 interaction points of the LHC at the CERN laboratory near Geneva, Switzerland. In 2010 and since March 2011 LHC has been colliding proton beams at the unprecedented centre of mass energy of $7 \mathrm{TeV}$. During the last month of 2010 operation was dedicated to $\mathrm{Pb}$-ion collisions at a centre of mass energy of $2.76 \mathrm{TeV}$ per nucleon pair. A challenging task of the previous months was to cope with the increasing event rates due to the increasing luminosity delivered by the LHC. A survey of the main ATLAS sub-detector systems, their operating conditions and the performance with colliding beams will be presented in this talk. The operation and results obtained from the data collected so far demonstrate that the detector is robust and functioning very well.
\end{abstract}

\section{Introduction}

The ATLAS detector [1] is one of the general purpose experiments installed at the Large Hadron Collider (LHC) at CERN. The ATLAS detector has been built and is maintained by a large collaboration of around 3200 scientists, (including $\sim 1000 \mathrm{PhD}$ students), from 174 institutions and 38 countries. It is designed to study processes at the $\mathrm{TeV}$ scale, search for the Higgs boson and for physics beyond the Standard Model as well as to make precision measurements of Standard Model processes. The ATLAS detector covers almost the whole solid angle around the collision point, see Figure 1 [1]. It is $\sim 46 \mathrm{~m}$ long and has a diameter of $24 \mathrm{~m}$. Its total weight is 7000 tons and is read out through a total of 88 million channels, which are connected by more than $3000 \mathrm{~km}$ of cables.

Pattern recognition, momentum and vertex measurements, and electron identification are achieved with a high-resolution semiconductor pixel and strip detectors (SCT) in the inner part of the tracking volume, and straw-tube tracking detectors (TRT) with the capability to generate and detect transition radiation in its outer part. This whole system was designed to achieve precise tracking and vertexing with transverse momentum $\sigma / p_{T}=0.038 \% p_{T}(\mathrm{GeV}) \oplus 1.5 \%\left(<2 \% p_{T}\right.$ $<35 \mathrm{GeV}$ ).

The superconducting solenoid is surrounded by an hermetic calorimeter that covers the pseudorapidity range $|\eta|<4$.9. A high granularity liquid-argon (LAr) electromagnetic sampling calorimeter, with excellent performance in terms of energy and position resolution $(\sigma / \mathrm{E} \sim 10 \% / \sqrt{E}(\mathrm{GeV}) \oplus 0.7 \%)$ covering $|\eta|<3.2$. The hadronic calorimetry in the range $|\eta|<1.7$ is provided by a scintillator-tile 
calorimeter. In the end-caps $(|\eta|>1.5)$, LAr technology is also used for the hadronic calorimeters. The LAr forward calorimeter provide both electromagnetic and hadronic energy measurements, and extends the pseudorapidity coverage to $|\eta|=4.9$. The hadronic energy resolution is $~$ $50 \% / \sqrt{E}(\mathrm{GeV}) \oplus 3 \%$ in the barrel region.

Outside the calorimeter, air-core toroids provide a magnetic field for the muon spectrometer up to $7.5 \mathrm{Tm}(\sim 1 \mathrm{~T}$ field at the center of each coil). Three sets of precision drift tubes (MDT) and cathode strip chambers (CSC) provide an accurate measurement of the muon track curvature in the region $|\eta|<2.7$. Resistive-plate (RPC) and thin-gap chambers (TGC) provide muon triggering capability up to $|\eta|<2.4$.

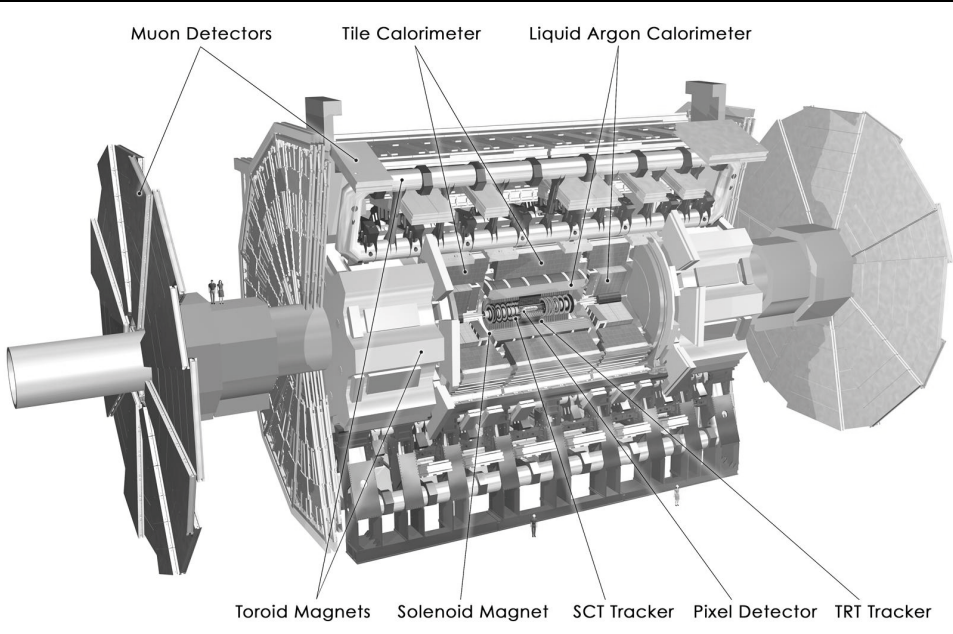

Figure 1. A schematic representation of the different components of the ATLAS detector.

The muon spectrometer provides efficient triggering, identification and momentum measurements. It was designed to measure muon momenta with a resolution of $4 \%$ for muons with $3 \mathrm{GeV}<p_{T}<100 \mathrm{GeV}$ and increasing to $10 \%$ at $1 \mathrm{TeV}$. The precision detectors provide $\sim 30-50 \mu \mathrm{m}$ tracking resolution up to $|\eta|<2.7$. The muon spectrometer defines the overall dimensions of the ATLAS detector.

Several detectors cover the forward regions on both sides of the ATLAS detector near the beam line, with the aim of measuring and monitoring the LHC luminosity, as well as providing physics measurements in the very forward regions. At $17 \mathrm{~m}$ from the interaction point there are luminosity monitor detectors based on Cherenkov tubes (LUCID), at $140 \mathrm{~m}$ there is a zero degree 
calorimeter for detecting photons and neutrons as can be produced in heavy Ion collisions, and at $240 \mathrm{~m}$ precision tracking detectors in Roman Pots will measure elastic scattering at very small angles for a total cross-section determination.

The trigger system has three levels, the first of which (L1) is fully hardware-based, and relies on information from the calorimeters and the Muon Spectrometer. The other two levels, the level two (L2) and the Event Filter (EF) are software based. The L2 trigger accepts data from defined Regions Of Interests (ROI) of L1 and the EF provides a full event reconstruction on computer farms. The 3 -stage trigger system reduces the $40 \mathrm{MHz}$ bunch crossing frequency to a LV1 trigger frequency of $75 \mathrm{kHz}$ and finally to a recording maximum frequency of $200-400 \mathrm{~Hz}$.

\section{Data Taking Operation, Luminosity and pile-up conditions}

From March to end September 2011 the LHC delivered pp collisions at a centerof-mass energy of $\sqrt{s}=7 \mathrm{TeV}$. The cumulative luminosity vs. time delivered by LHC, and that recorded by ATLAS are shown in Figure 2 left. The total recorded integrated luminosity is equal to $3.78 \mathrm{fb}^{-1}$ and the overall ATLAS data taking efficiency is $94 \%$. All the sub-detectors are operating with a very high efficiency $(>96 \%)$. The instantaneous peak luminosity continues to raise, and a value of $3.3 \times 10^{33} \mathrm{~cm}^{-2} \mathrm{~s}^{-1}$ was achieved in September. The average pile-up during the 2011 running is $\sim 12$ interactions per beam crossing, and is growing continuously, as is seen in Figure 2 right. This is one of the biggest challenges in the detector performance in 2011, but well under control. Currently, the systematic uncertainty of the luminosity measurement is 3.7\% [2], dominated by the uncertainty in the beam current. Van der Meyer scans are being taken to reduce further this error.

The ATLAS computing infrastructure consists of $\sim 70$ sites, organized in a hierarchical structure ("tiers"). It includes the CERN computing center (Tier-0), 10 big regional centers (Tier-1), and various smaller sites (Tier-2). During datataking, the raw data is processed within $36 \mathrm{~h}$ to do first calibrations, data quality checks and mask bad cells. Data are usable for physics analysis $\sim 1$ week after data taking. ATLAS distribute $>800000$ processing jobs/day with a peak rate of $10 \mathrm{~GB} / \mathrm{s}$. Since the start of LHC ATLAS distributed more than 66 Petabytes (PB) of data all over the globe, to allow the worldwide collaboration to do analysis efficiently. 

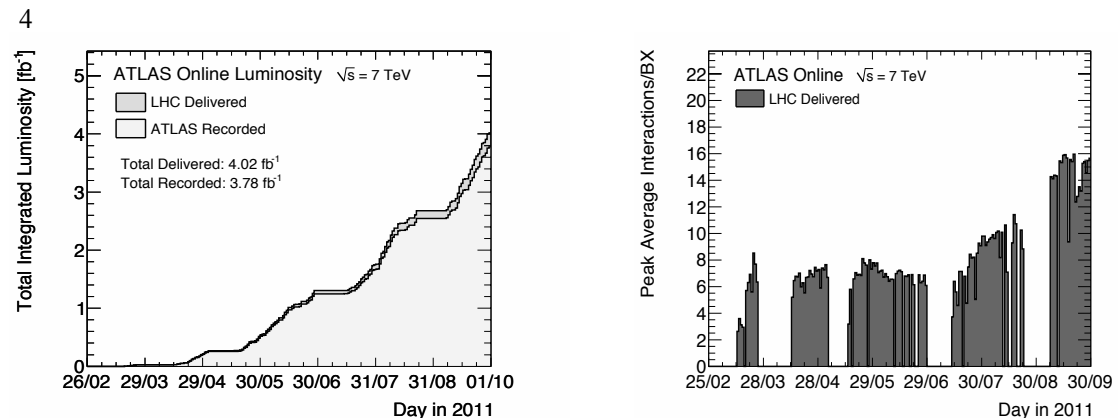

Figure 2. Left: Cumulative Luminosity (delivered by LHC and recorded by ATLAS) versus time during pp collisions at $\sqrt{\mathrm{s}}=7 \mathrm{TeV}$. Right: The peak average "events per beam crossing" versus time in 2011.

\section{Inner tracker performance}

The Pixels, SCT and TRT are operating very efficiently and providing high quality data for the physics analysis. A significant effort is put in the precise positioning of the hits recorded by the sensors, to understand the material inside the tracking volume, pattern recognition and track fitting, detector alignment and particle identification. The residuals in the most precise direction of the pixel SCT and TRT sub-detectors are respectively $9 \mu \mathrm{m}[\mathrm{r} \phi], 25 \mu \mathrm{m}[\mathrm{r} \phi]$ and $118 \mu \mathrm{m} / \mathrm{straw}$, with the autumn 2010 alignment. This is in close agreement with the $\mathrm{MC}$ residuals obtained for a perfect alignment, reaching values very close to design. The achieved primary vertex $x$ resolution is $\approx 20 \mu \mathrm{m}$. Figure 3 shows the reconstructed invariant mass of the $Z \rightarrow \mu^{+} \mu^{-}$comparing spring and summer 2011 alignment and comparing with Monte Carlo (MC) simulations, which were made assuming a perfectly aligned detector. The summer 2011 alignment includes several improvements, in particular a better alignment of ID with respect to the solenoid B field. The improvement is clearly visible and in good agreement with MC. This is also visible in Figure 3 right, in which the $\mathrm{Z}$ mass $\phi$ modulation has disappeared with new alignment corrections. Measurements of the properties of well studied particles, such as the $\mathrm{K}^{0} \mathrm{~s}, \varphi, \mathrm{D}, \Omega, \Xi, \Lambda$, and $\mathrm{Z}$, have allowed the momentum scale to be determined to around one part for thousand for lower momentum $(<20 \mathrm{GeV})$ and to one part per hundred at higher momentum (up to $\sim 100 \mathrm{GeV}$ ) [3]. The resolution was found as expected to be dominated by multiple scattering in the low $p_{T}$ region $\left(<2 \%\right.$ at $\left.p_{T}<35 \mathrm{GeV}\right)$. 

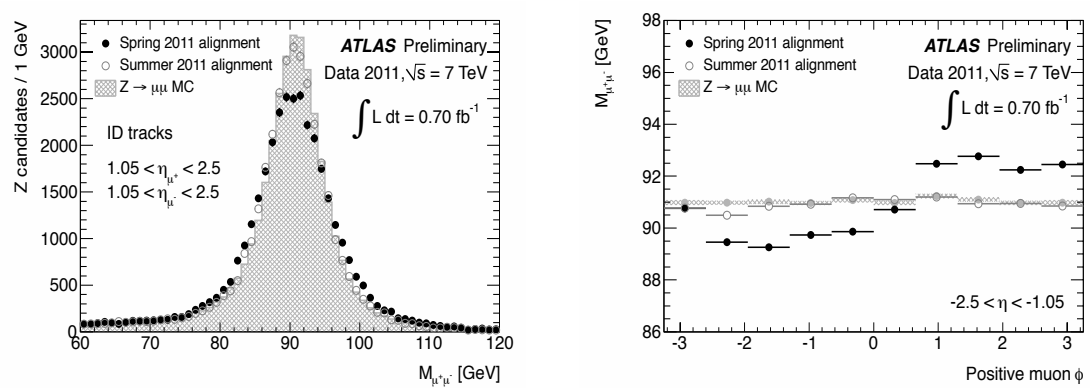

Figure 3. Left: Reconstructed invariant mass distribution of $Z \rightarrow \mu+\mu$ - candidates. Right: Mean $Z$ invariant mass versus $\varphi$. The data were processed using alignment information known in spring and summer 2011. MC simulations, which assume a perfect alignment, are also shown. The improvement of the summer 2011 alignment is clearly seen in both figures.

\section{Calorimeter, Jet energy scale and $E_{T}$ miss performance}

The validation of the em LAr calorimeter performance in terms of calibration, resolution and linearity was done using well-studied particle decays such as those of $\pi^{\circ}, J / \Psi, Z$, see Figure 4 . The calibration was tuned to describe the $\mathrm{Z} \rightarrow \mathrm{e}^{+} \mathrm{e}^{-}$ resonance. The data are compared with the Monte Carlo showing a good agreement, which constitutes a proof of the excellent calibration and response linearity. The linearity is $\sim 0.3-1.6 \%$ up to $1 \mathrm{TeV}$ for $|\eta|<2.47$. The constant term of the em energy resolution is $\sim 1.2 \%$ in the barrel, $1.8 \%$ in the end-cap and $2.5 \%$ in the forward calorimeter [4]. More calibrations will bring these soon to the design target of $0.7 \%$. In 2011 significant improvements were implemented in the relative alignment of the em calorimeter with respect to the inner detector.
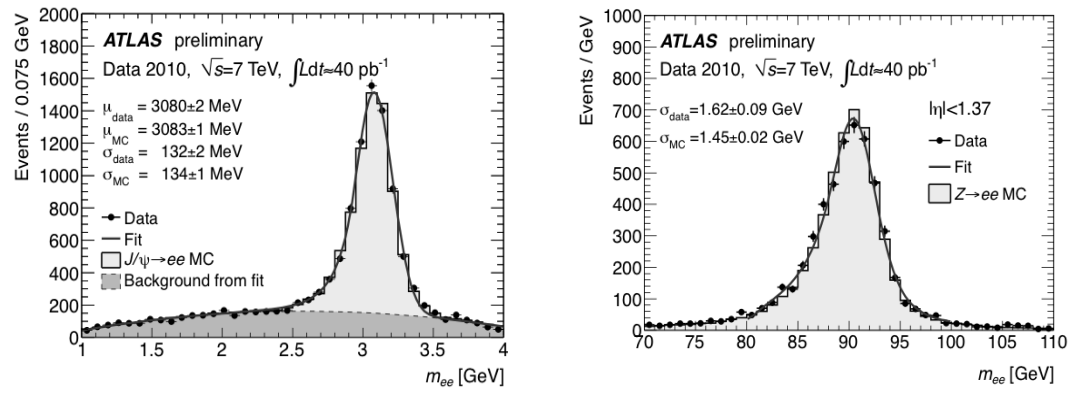

Figure 4. Invariant mass spectrum for electron pairs for $\mathrm{J} / \Psi$ and $\mathrm{Z}$ decays: left and right plots respectively. A good agreement with the Monte Carlo is observed. 
This is illustrated in Figure 5 left, showing the cluster-track matching variables used in the electron and photon reconstruction and identification before and after the alignment (black and red points respectively). After alignment there is a good agreement with $\mathrm{MC}$ using $\mathrm{W} / \mathrm{Z}->\mathrm{ev} / \mathrm{e}^{+} \mathrm{e}^{-}$events. Figure 5 right shows the reconstruction efficiency measured using $\mathrm{W} \rightarrow \mathrm{e} v$ events and predicted by $\mathrm{MC}$ as a function of $\eta$. The reconstruction $\varepsilon$ precision is within $\pm 1 \%\left(p_{T}>35 \mathrm{GeV}\right)$.

The performance of the hadron calorimeter is important for many physics analyses at the LHC involving jets and missing $E_{T}$, such as Higgs, SUSY, etc. The validation of the calibration of the Tile calorimeter at the electromagnetic scale (extracted in the testbeam for $11 \%$ of the modules) was obtained with cosmic rays, muons from collisions and using in-situ calibration method (e/p).
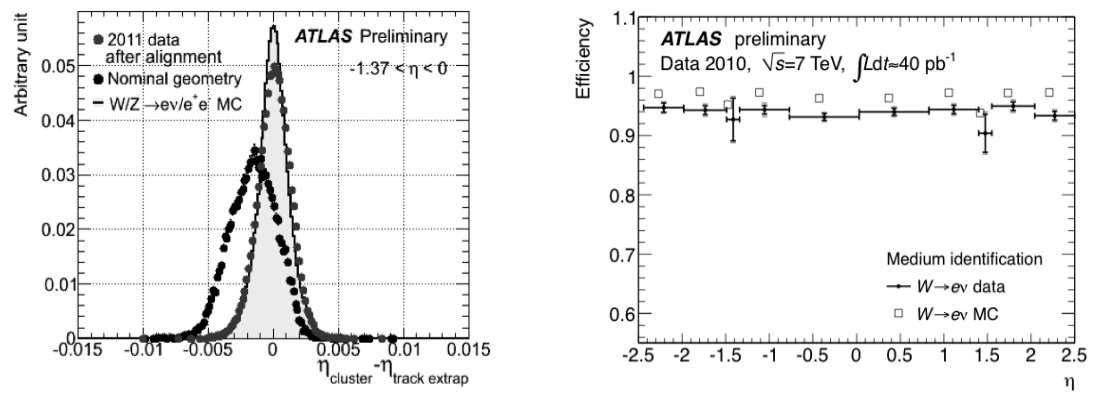

Figure 5 Left: Improvements brought by the relative alignment of the em calorimeter and the ID in 2011 to the cluster-track matching variables used in the $\mathrm{e}^{-}$and $\gamma$ reconstruction and identification. Right: Efficiencies measured using $\mathrm{W} \rightarrow \mathrm{e} v$ events and predicted by $\mathrm{MC}$ as a function of $\eta$.

Figure 6 left shows the mean value of the ratio between the energy deposited in the Tile calorimeter and the track momentum measured by the ID as a function of $\eta$, showing a very good agreement with MC. Figure 6 right shows the jet resolution in the barrel region with different calibration methods using 2010 data. The resolution is approaching design and the agreement between data and $\mathrm{MC}$ is within $10 \%$. The constant term extrapolated to $2 \mathrm{TeV}$ is estimated to $\sim 3 \%$ with $\mathrm{GCW} / \mathrm{LCW} / \mathrm{GS}$ calibrations. In 2011 the presence of pile-up worsens the low $p_{T}$ resolution by $\sim 20 \%$. Improvements are expected after careful pile-up corrections for in-time/out-time bunches, noise threshold tuning in LAr calorimeter and using tracking information. 

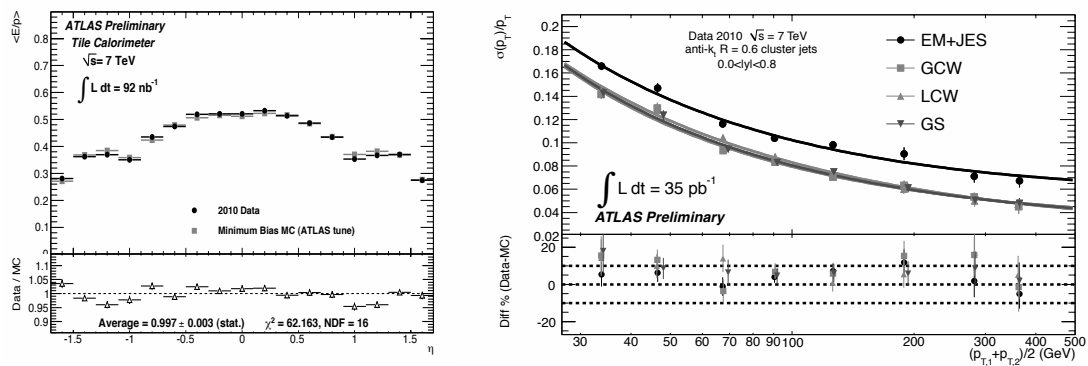

Figure 6. Mean value of the ratio of the e/p ratio in the Tile calorimeter vs h. Right: Jet resolution as a function of the momentum for different calibration schemes.

The jet energy scale error $(\triangle \mathrm{JES})$ is the main uncertainty in many physics results (jet/dijet cross section, top, etc). It was evaluated on data for jets with 20 $\mathrm{GeV}<p_{T}<3.5 \mathrm{TeV}$ and $|\eta|<4.5$ [5]. It was set using the "EM+JES" calibration method (em scale+MC corrections for non compensation + dead material). In 2010 the $\triangle \mathrm{JES}$ was $\sim 2.5 \%$ for $60<p_{T}<800 \mathrm{GeV}|\eta|<2.1$ up to $7 \% \max$ in the forward region as seen in Figure 7 left. The validation of the jet energy scale was done with in situ techniques ( $\gamma+$ jet, multi-jet, track-jet) up to jet $p_{T} \sim 1 \mathrm{TeV}$ showing agreement between data and MC within the errors, see Figure 7 right. In 2011 the pile-up worsened $\triangle \mathrm{JES}$, it's contribution $\left(\sim 5 \%\right.$ at $p_{T}=20 \mathrm{GeV}, \sim 0 \%$ $>100 \mathrm{GeV})$ has to be added in quadrature with the $2010 \triangle \mathrm{JES}$. As for the resolution improvements are expected after careful pile-up corrections for intime/out-of-time bunches and noise threshold tuning in the LAr calorimeter. An increase of statistics in 2011 will allow the use of in situ methods $(\gamma+$ jet, multijet, track-jet) for recalibration and further improvement of the jet scale precision. The goal is to achieve $1 \%$.
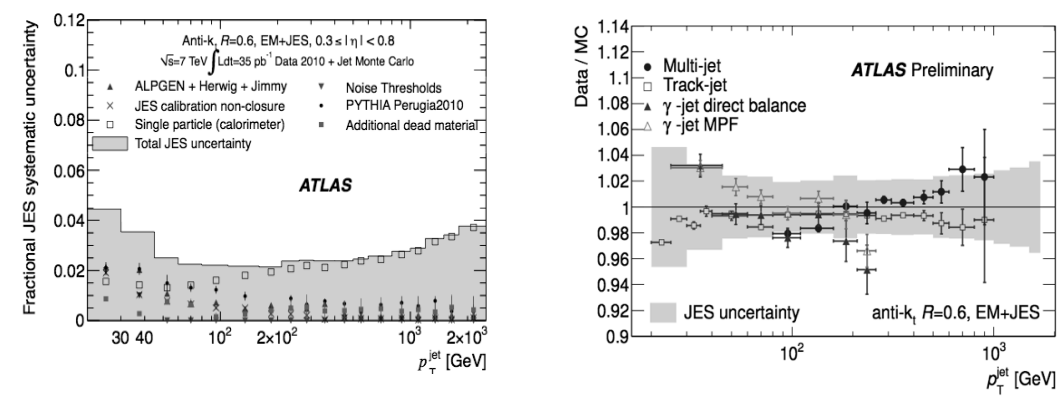

Figure 7 Left: Fractional jet energy scale systematic uncertainty as a function of the jet $p_{T}$ for jets in the barrel calorimeter $(0 .<|\eta|<0.8)$. The total uncertainty is shown as the solid gray area. Right: Data to Monte Carlo simulation ratios for several in-situ techniques that test the jet energy scale. 
The calorimeters also contribute to the measurement of the missing transverse energy ( $\left.E_{T \text { miss }}\right)$, a quantity sensitive to the calorimeter performance in terms of noise dead cells and mis-calibration. It has been measured in the ATLAS detector with the $7 \mathrm{TeV}$ collision $\mathrm{p}-\mathrm{p}$ and $\mathrm{Pb}-\mathrm{Pb}$ data [6] and found to be in good agreement with the simulation, see Figure 8 left. The $E_{T}$ miss resolution in $\mathrm{p}$ and $\mathrm{Pb}$ collisions is $\sim 48 \% \sqrt{ } \sum E_{T}$. Figure 8 right shows the distribution of the sum $E_{T}$ as measured in a data sample of di-jet events selecting two jets with $p_{T}>25 \mathrm{GeV}$. The expectation from MC simulation, normalized to the number of events in data, is superimposed showing once more a good agreement.
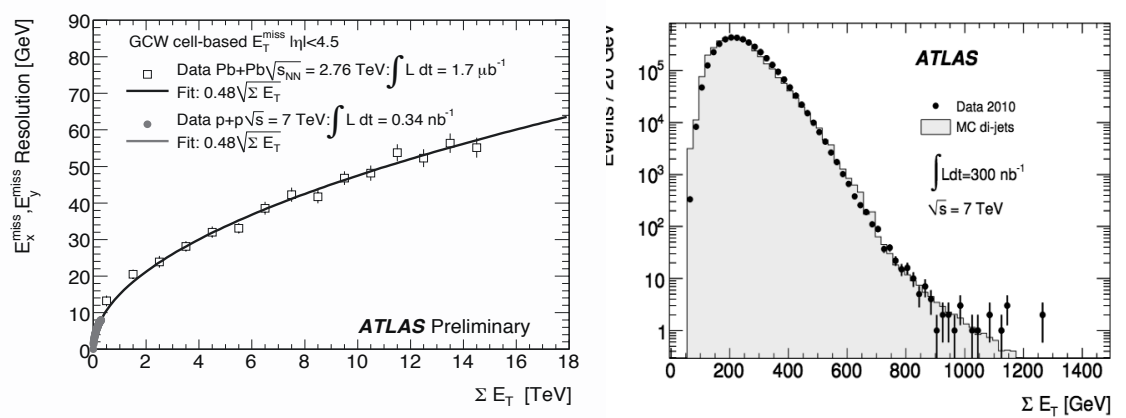

Figure 8 Left $E_{T}$ miss resolution versus the total transverse energy for minimum bias data in the pp and $\mathrm{Pb}-\mathrm{Pb}$ collisions data. Right: Distribution of $\sum E_{T}$ as measured in a data sample of di-jet events selecting two jets with $p_{T}>25 \mathrm{GeV}$. The expectation from MC simulation is superimposed.

\section{Muon spectrometer performance}

Figure 9 left shows the muon spectrometer alignment resolution (in sector 7) as a function of $\eta$. The barrel achieves a resolution of $50 \mu \mathrm{m}$, close to the design goal, whereas the end-cap gives a resolution of around $110 \mu \mathrm{m}$ indicating that further improvements are necessary. Further calibration runs with collision data and with magnetic field off will be needed to achieve the desired performance. Figure 9 right shows the muon reconstruction efficiency as a function of $p_{T} ; 97 \%$ is achieved for $p_{T}>20 \mathrm{GeV}$ in good agreement with MC. 

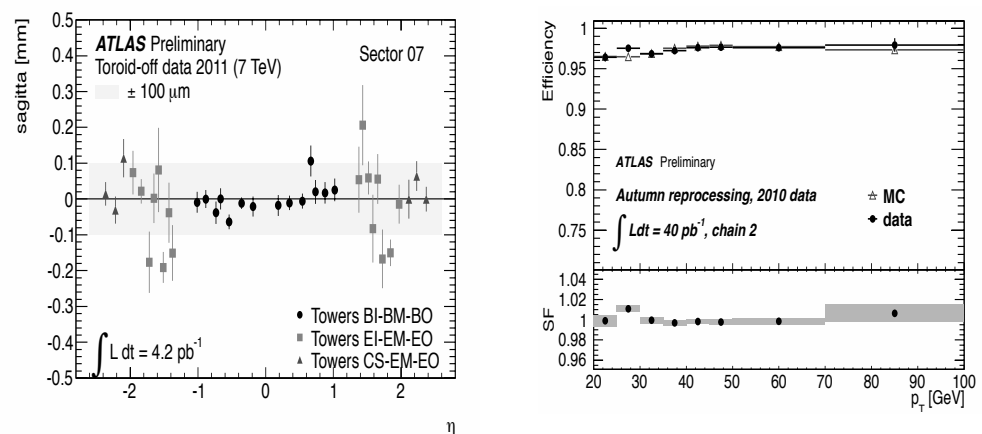

Figure 9 Left: Muon spectrometer alignment resolution as a function of $\eta$ in sector 7 (black points for the barrel and the red and blue corresponding to the end-caps). Right: Muon reconstruction efficiency as a function of $p_{T}$.

The muon spectrometer performance was checked with well know resonance particles decaying into a pair of muons as illustrated in Figure 10 for $\mathrm{J} / \Psi$ and $\mathrm{Z}$ decays. The achieved mass resolution is $2.2 \%$ at $91 \mathrm{GeV}, 1.9 \%$ at $\sim 3$ $\mathrm{GeV}$, close to design expectations. The response is linear and the absolute momentum scale is known to $\sim 0.2 \%[7,8]$.
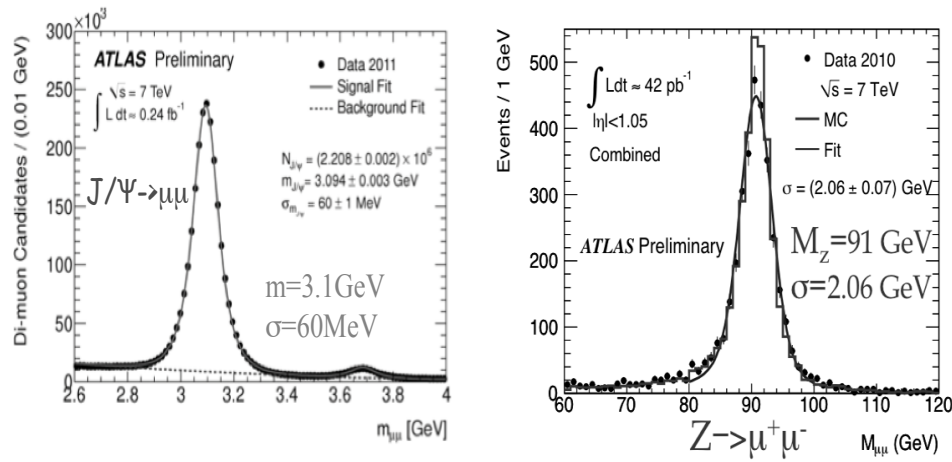

Figure 10. Di-muon invariant mass in data and $\mathrm{MC}$ from $\mathrm{J} / \mathrm{psi}$ and $\mathrm{Z}$ decays.

\section{Trigger performance}

The trigger selection is defined by a trigger menu which consists of more than 300 individual trigger signatures, such as electrons, muons, particle jets, etc. The composition of the trigger menu depends on the instantaneous LHC luminosity, the experiment's goals for the recorded data, and the limits imposed by the available computing power, network bandwidth and storage space. The trigger rates for various levels and data streams agree very well with 
expectations and scale linearly with luminosity over a wide range of luminosity as shown in Figure 11, giving us reliable extrapolation for higher luminosity/pile-up. Triggers thresholds are tuned such that we keep a major trigger in a physics analysis un-prescaled while the output trigger rate is kept at $\max$ of 300-400 MHz. The good performance of the trigger system can be shown in Figure 12 in steeply rising turn-on trigger efficiency curves saturating at high values for jets, muon, electron and taus.
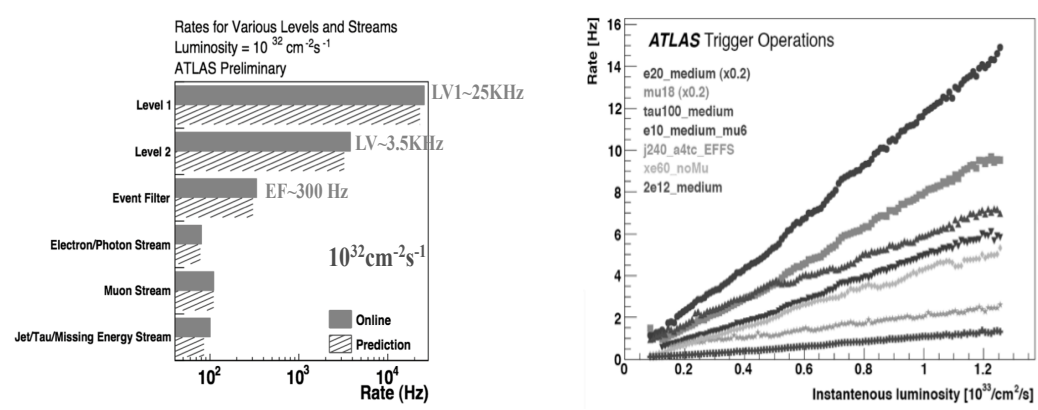

Figure 11 Left: measured and expected rates for LV1,2,EF and physics streams $\left(\mathrm{e} / \gamma, \mu, \mathrm{jet} / \tau / \mathrm{ET}_{\text {miss }}\right)$. Right: Rates of various physics streams as a function of the instantaneous luminosity.
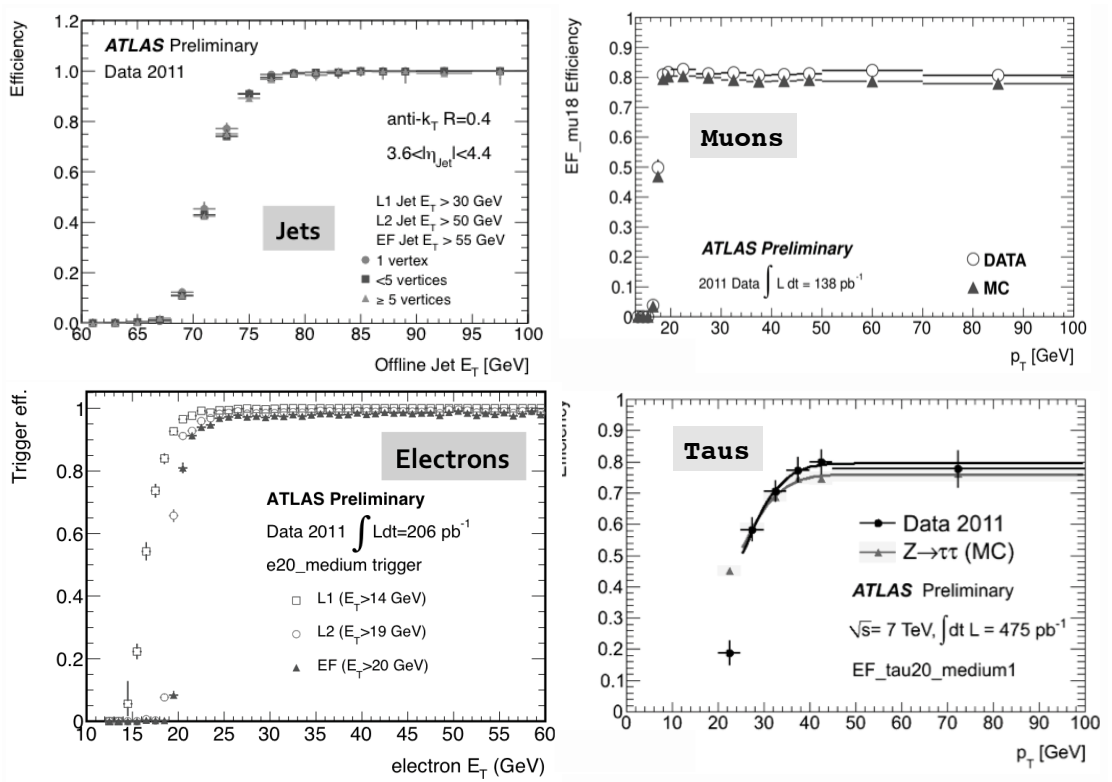

Figure 12. Examples of trigger efficiencies for jets, muon, electron and taus. 


\section{Conclusions}

ATLAS successfully recorded $3.78 \mathrm{fb}^{-1}$ with an efficiency of $\sim 94 \%$ from data acquisition/processing to use in data analysis. The detector is performing very well, data is well described by the simulation and approaching design performance. The continuously increasing contribution of pile-up in 2011 is a big challenge but is under control. ATLAS is now exploiting the full physics potential of LHC. 73 papers on detector performance and physics results using collision data have been published.

\section{Acknowledgments}

These achievements were only possible thanks to the outstanding performance of the LHC machine team and to the many years of dedicated work of the ATLAS collaboration in test beam activities, MC tuning and intense commissioning of the ATLAS detector well before the data taking started, with cosmics rays and now with collision data, in parallel with intense physics programme.

\section{References}

1. The ATLAS Collaboration [G. Aad et al.], The ATLAS Experiment at the CERN Large Hadron Collider, JINST 3:S08003, 2008.

2. ATLAS Collaboration, G. Aad et al., Updated Luminosity Determination in pp Collisions at $\sqrt{\mathrm{s}}_{\mathrm{s}}=7 \mathrm{TeV}$ using the ATLAS detector, ATLAS-CONF-2011-011.

3. ATLAS Collaboration, G. Aad et al., Kinematic Distributions of K0s and $\Lambda 0$ decays in collision data at $\sqrt{\mathrm{s}}_{\mathrm{s}}=7 \mathrm{TeV}$, ATLAS-CONF-2010-033.

4. ATLAS collaboration, Expected electron performance in the ATLAS experiment, ATLAS-PHYS-PUB-2011-006

5. ATLAS Collaboration, G. Aad et al., Jet energy scale and its systematic uncertainty in proton-proton collision at $\sqrt{s}_{\mathrm{s}}=7 \mathrm{TeV}$ with ATLAS 2010 data, ATLAS-CONF-2011-032.

6. ATLAS Collaboration, G. Aad et al., Performance of the Missing transverse Energy Reconstruciton and Calibration in Proton-Proton Collisions at a Centre-of-Mass Energy of $\sqrt{S}_{\mathrm{s}}=7 \mathrm{TeV}$ with the ATLAS Detector, ATLAS-CONF-2010-057

7. ATLAS Collaboration, G. Aad et al., A measurement of the ATLAS muon reconstruction and trigger efficiency using $\mathrm{J} / \psi$ decays, ATLAS-CONF-2011-021.

8. ATLASCollaboration,G.Aadetal.,Determination of the muon reconstruction efficiency in ATLAS at the $\mathrm{Z}$ resonance in p-p collisions at $\sqrt{\mathrm{s}}_{\mathrm{s}}=7 \mathrm{TeV}$, ATLAS-CONF-2011-008. 